\title{
Filigrane
}

Écoutes psychothérapiques

\section{Vivre l'engagement et le contre-transfert en société précaire}

\section{Jean Furtos}

Volume 21, numéro 1, printemps 2012

Psychanalyse et engagement

URI : https://id.erudit.org/iderudit/1012880ar

DOI : https://doi.org/10.7202/1012880ar

Aller au sommaire du numéro

Éditeur(s)

Revue Santé mentale au Québec

ISSN

1192-1412 (imprimé)

1911-4656 (numérique)

Découvrir la revue

Citer cet article

Furtos, J. (2012). Vivre l'engagement et le contre-transfert en société précaire. Filigrane, 21(1), 31-38. https://doi.org/10.7202/1012880ar
Résumé de l'article

L'engagement, c'est se donner en gage dans une relation interhumaine, thérapeutique ou pas. C'est accepter d'être dérangé par autrui souffrant, dérangement dont le couple tranfert/contre-tranfert est l'un des paradigmes. L'auteur explore ce rapprochement à travers la manière dont il a été dérangé par la souffrance psychique d'origine sociale dans les années quatre-vingt-dix, à travers également la clinique rapprochée avec les personnes en précarité. Cela implique un mode d'être à la fois proximal et distancié. 


\title{
Vivre l'engagement et le contre-transfert en société précaire
}

\author{
Jean Furtos
}

L'engagement, c'est se donner en gage dans une relation interhumaine, thérapeutique ou pas. C'est accepter d'être dérangé par autrui souffrant, dérangement dont le couple tranfert/contre-tranfert est l'un des paradigmes. L'auteur explore ce rapprochement à travers la manière dont il a été dérangé par la souffrance psychique d'origine sociale dans les années quatre-vingt-dix, à travers également la clinique rapprochée avec les personnes en précarité. Cela implique un mode d'être à la fois proximal et distancié.

" uand on s'engage, on ne sait pas à quoi on s'engage» écrivait Emmanuel Levinas (1978).

Sait-on à quoi l'on s'engage lorsqu'on prend un patient en thérapie, en analyse, lorsqu'on s'engage dans un métier, dans la cité, sur le plan personnel et intime, comme lorsqu'on décide de faire un enfant? Le savons-nous vraiment? Heureusement non! Sinon, peut-être ne nous engagerions nous jamais, car il faut une dose certaine d'idéalisation et d'inconscience (parfois de masochisme...) pour s'engager, se donner en gage, ou tout au moins donner une partie de soi, de son temps, de sa vie, en gage.

Ce qui est proposé à l'analyste dans l'expérience psychanalytique, via le transfert, c'est d'accepter d'être affecté au niveau de sa vie psychique propre, au point de porter l'autre en soi, comme un malaise, une séduction, une question, en tout cas une altérité énigmatique. Si le contre-transfert est l'outil principal de l'analyste, cela nous renvoie à l'engagement risqué qui consiste d'abord à ne plus savoir si le contre-transfert appartient à l'autre ou à soi, à être entre-deux et en même temps terriblement intériorisé. Accepter d'être dérangé par l'autre est au fondement de toute relation intersubjective et donc, de la relation thérapeutique. J'ajoute qu'il s'agit d'être dérangé au service de la clinique, pour le «bien» d'autrui, car on ne peut nier, en tout cas selon moi, que si nous faisons ce métier, c'est tout de même, au-delà de l'ambivalence, pour aider les autres à aller mieux dans leurs difficultés psychiques, 
dans leur vie. C'est accepter d'avoir un dérangement interne qui exige de nous une élaboration, une réflexivité, un mécanisme intermédiaire entre appropriation et désappropriation, entre ce qui nous appartient et ce qui appartient à l'autre; c'est à la fois accepter d'être squatté par l'autre, quelquefois littéralement et non métaphoriquement, ou à l'inverse, accepter d'être délogé de nous-mêmes et de toute certitude.

La neutralité, ladite neutralité en psychanalyse, n'est certainement pas l'indifférence car elle est supposée bienveillante, et bienveillance $=$ vouloir $d u$ bien, malgré l'ambivalence inhérente à toute relation. J'ai toujours pensé, avec quelques collègues, que nous sommes obligés d'aimer nos patients pour les aider à vivre; mais aimer, dans ce contexte, signifie aimer la vie de l'autre, aimer que l'autre vive dans sa pensée, dans sa parole, dans ses actions, dans ses engagements personnels, dans la manière dont il se situe ou pas dans la suite des générations. Si l'on n'aime pas du tout la vie de l'autre, autant passer la main! Cet amour (réciproque) dit « de transfert» entraine souffrance, et exigence intellectuelle et affective. Mais lorsqu'advient une brèche dans la répétition, une ouverture pour une vie autre, une vie nouvelle, un commencement, alors quelque chose de l'ordre de la joie peut être éprouvé et partagé.

J'ai été confronté à une forme de transfert décalé et collectif dans les années 1993-1994, époque à laquelle je me suis orienté vers de nouvelles modalités de la souffrance contemporaine en rapport avec la précarité, c'està-dire, en rapport avec le couple confiance/méfiance face à la précarité et l'incertitude du monde: avec une triple confiance ou une triple méfiance en l'autre, en soi-même, et en l'avenir. À cet égard, nous sommes un certain nombre à avoir été confrontés à ce que Freud avait nommé la souffrance psychique d'origine sociale: une sacrée affaire dans la culture qui est la nôtre. Cette souffrance psychique d'origine sociale avait déjà été théorisée par Freud dans Malaise dans la culture. À notre époque, elle fait partie des discussions de café, autour de la question des délocalisations, de la précarité des droits acquis, de l'hyperindividualisme contemporain, de l'emprise financière de la phase néolibérale du capitalisme qu'on n'aurait jamais pu imaginer d'une cruauté aussi froide.

La toute première constatation clinique est qu'il y a en effet une souffrance psychique d'origine sociale chez les plus démunis, dont les gens de la rue, les itinérants, les chômeurs de longue durée, les demandeurs d'asile déboutés de leurs demandes, etc. Mais plus nous avancions dans la compréhension de cette détresse, plus nous constations cliniquement que ce qui était 
vrai au niveau de ceux qui sont soi-disant à la marge de la société, était vrai aussi pour ceux qui sont au centre, au cœur de la société. On ne sait jamais suffisamment, quand on travaille avec la marge, que l'on travaille aussi pour le centre, même si le centre n'en veut rien savoir. Le centre fonctionne en effet projectivement pour éviter de vivre un dérangement personnel. C'est l'autre qui doit poser problème, l'homme de la rue, l'étranger ou le «fou dangereux» (celui qui a des problèmes, des soucis identitaires et qui nous dérange), jusqu'à ce que l'on s'aperçoive, à travers par exemple les réflexes néonationalistes, qu'un grand nombre de citoyens se sentent atteints dans leur identité et leur intégration sociale, même s'ils peuvent encore gagner suffisamment d'argent pour élever leur famille, prendre des vacances et se faire de petits plaisirs. Fait important, le malaise térébrant de la phase actuelle de la modernité est une souffrance psychique qui ne peut souvent être reconnue que par autrui, surtout dans ses formes extrêmes, et c'est là que nous en revenons au contre-transfert.

J'ai suggéré en 2001 lors d'un séminaire national de recherche clinique au monastère le Corbusier à l'Arbresle, près de Lyon ${ }^{1}$, que la souffrance psychique est un contre-transfert des praticiens du soin psychique et/ou social. À l'époque, René Roussillon m'avait fortement encouragé à poursuivre ma réflexion dans ce sens. Force est de constater qu'effectivement, dans les pays où il n'y a pas d'État-providence à minima, pas de welfare, pas de care, pas de travail social, pas de République ou de Monarchie constitutionnelle qui se soucie de ceux qui sont en difficulté psychosociale, la souffrance psychique d'origine sociale n'est pas vécue comme telle. Il y a simplement des épisodes de soumission et de passivité, entrecoupés de violences paroxystiques contre soi ou contre autrui, qui surprennent toujours ceux qui ne voulaient pas en entendre parler.

À partir de 1999, j'ai décrit la clinique psychosociale comme «la prise en compte de la souffrance psychique qui apparait sur les lieux du social ${ }^{2} », c^{\prime}$ està-dire bien sûr dans la rue, mais aussi dans les milieux du travail social, dans les lieux d'enseignement et du travail. Nous constatons maintenant que les médecins, les juges, les cadres supérieurs peuvent être sujets à une souffrance psychique d'origine sociale qui n'est remarquée que si elle affecte quelqu'un d'autre qui en accepte le transfert, c'est-à-dire qui accepte d'être affecté par cette souffrance, de la porter en soi un temps. C'est l'une des caractéristiques les plus remarquables de cette souffrance que d'être portée par quelqu'un en face ou à côté, qui accepte d'en être affecté. Souffrir seul n'est pas bon pour la santé. La perception de cette souffrance prend le sens d'une exclusion : «Je 
ne suis plus souhaité sur la scène sociale, je ne peux plus y apparaître par la parole et par l'action. » Celui ou celle qui pense ce vécu d'exclusion a la potentialité de refuser cette pensée, de rentrer dans le déni et le clivage, dans ce que j'ai appelé le syndrome d'auto-exclusion (Furtos, 2008). Seul celui qui est en face ou à côté, par le malaise qu'il ressent, peut témoigner de la souffrance de l'autre, inappropriable pour lui à ce moment-là. Celui ou celle qui ressent ce malaise est alors porté par une sorte de rêverie maternelle primaire: tous les éléments bêta sont déposés dans la psyché de l'autre en vue d'être élaborés, psychisés, transformés en éléments alpha pour être remis dans la circulation intersubjective, et quelque fois réappropriés par l'autre par la parole, par les actes, par les rituels. Un certain nombre de personnes travaillant dans la clinique psychosociale, et pas seulement les psy, ont l'éthique d'accepter ce malaise et d'en faire quelque chose sur le plan professionnel en en parlant avec leurs pairs, leur hiérarchie, dans l'analyse de leur pratique, en supervision. En faire quelque chose de telle manière que ce transfert, qui n'est pas d'ordre psychanalytique dans la mesure où il est vécu en dehors d'une relation analytique, puisse être élaboré au service de l'autre, que le malaisesouffrance déposé dans les gens ne soit pas un trou noir soumis à l'indifférence, au blindage, au refus d'être dérangé.

J'ose affirmer que le transfert est la capacité d'accepter le dérangement nécessairement produit par l'autre par le seul fait qu'il existe dans son histoire; la psychanalyse est la professionnalisation maximale de cette capacité, dans un contrat très particulier, dans une alliance thérapeutique très particulière. Plusieurs psychanalystes en France et ailleurs reçoivent des gens démunis, en grande précarité sociale, qui peuvent même être parfois des cadres supérieurs. En France, ils ont fondé les CPCT: Centres de psychanalyse et de consultation thérapeutique. Il s'agit d'analystes issus de l'école de la Cause freudienne qui ont constaté la souffrance psychique d'origine sociale et qui ont postulé qu'un certain nombre de nos concitoyens pouvaient avoir besoin de rencontrer un analyste quelques temps sans pour autant s'engager dans une analyse, et d'entamer une relation qui passe par la parole et la reconnaissance d'existence, la reconnaissance d'un malaise, celui de la disparition de soimême comme ayant une place par la parole et par l'action sur la scène sociale (Furtos, 2008).

Dans les expériences et les rencontres qui se déroulent aux CPCT et ailleurs, le principe de départ est sans doute, comme l'écrit Jacqueline Dheret (2011, p. 6, 8), 
de faire de la souffrance humaine une affaire d'éthique et non de pathologie. [...] Le délitement actuel du lien social, la difficulté rencontrée dans le rapport à l'autre, le statut de la parole aujourd'hui, toujours au service du bien-être, nous conduisent à tempérer la notion de demande. La formation du mythe du sujet autonome implique, en effet, une dégradation du statut de la parole. Une parole qui ne se supporterait plus du malentendu, qui ne serait plus infiltrée de satisfaction, mais qui produirait des effets de désignation en série. Avec, en contrepoint, une invitation à faire du rapport de chacun à sa vie, à son travail, à sa maladie, etc., un «storry telling» permanent. [...] C'est le paradoxe que nous traitons: celui d'inviter à venir nous voir, pour recréer, dans l'espace public de la cité, l'intimité d'une parole mesurée qui concerne le sujet et l'engage, sans le confronter au vide ou au «tout dire». La notion de précarité recouvre des réalités diverses, aussi bien sociales que psychiques. Or, la réalité sociale, c'est la réalité psychique: quand le malaise de chacun ne trouve plus à se loger dans ce que la civilisation propose, la pulsion de mort reprend ses droits. [...] Il nous faut donc tenir compte de ce qui se généralise, pour avoir une chance d' «attraper » ce qui déborde la personne et ne s'adresse à personne.

Je voudrais à ce point aborder la question de l'orthodoxie. On croit souvent qu'être orthodoxe, c'est suivre quasiment aveuglement les écrits des maîtres, des pionniers, comme l'ont été Freud, Lacan, Mélanie Klein et Bion pour ne citer que ceux-ci, et l'on oublie que tous ces maîtres au moment de ces écrits, étaient en position de transgression par rapport aux dogmes dominants, y compris au sein de l'Association psychanalytique internationale. Je propose donc de considérer la transgression non pas dans sa relation à un interdit, mais dans son sens étymologique, comme le fait d'aller plus loin, d'aller au-delà de ce qui est admis par l'opinion commune. À cet égard, l'engagement d'un analyste ou d'un psy, c'est d'abord de transgresser, c'est-à-dire d'aller au-delà de ce qu'on lui a appris, en fonction de ce qu'il estime être une pratique juste dans l'ici et maintenant. J'ai écrit sur ce thème dans un numéro de Rhizome, et je n'y reviendrai pas, sauf pour rappeler ceci :

Nos collègues médecins du travail universitaires et ergonomes nous ont appris depuis longtemps que l'écart entre ce qui est prescrit et ce qui est effectué dans le travail concret ne donne pas de bons résultats s'il n'est pas traité efficacement par «le travailleur», autant pour creuser un trou que pour travailler dans une usine nucléaire ou dans une administration; en 
clair, il y a toujours une différence entre ce que l'on doit faire, en principe, et ce à quoi l'on est confronté sur le terrain, si bien que le travailleur, quelle que soit sa compétence et sa responsabilité, se doit, s'il tient à un travail bien fait, de corriger l'écart par une initiative personnelle pour laquelle il pourra être blâmé ou félicité, bien que l'initiative reste le plus souvent ignorée de sa hiérarchie.

La formule générale du travail intelligent est $\mathrm{Te}=\mathrm{Tp}+\mathrm{e}$, dans laquelle $\mathrm{Te}$ est le travail effectué, Tp le travail prescrit, et e l'effort personnel du sujet pour s'adapter au terrain en fonction des besoins, de son implication personnelle, de la mise en ouvre de ce qu'il est, sa chair, en quelque sorte. (Furtos, 2006, p. 57)

Ce petit «e», c'est l'engagement du professionnel, sa capacité à mettre de soi dans le travail pour qu'il soit bien fait, et qu'il soit fait par un être vivant avec d'autres êtres vivants.

J'ai en tête beaucoup d'exemples où ce que j'ai fait était résolument « inorthodoxe » et venait d'une petite voix à l'intérieur de moi qui me disait : «tiens, je vais faire ceci», où "non, tu ne vas surtout pas faire cela», que ce soit pour entreprendre le défrichage d'un champ théorique et pratique encore inexploré, ou dans la difficile présence avec un patient où la seule manière d'être juste, c'est d'inventer, d'improviser, d'être vivant; c'est cela l'engagement.

J'en donnerai un exemple pour terminer.

Un fait clinique récent. Un de mes patients me téléphone un matin à l'hôpital: il a besoin d'un certificat urgent. Le rendez-vous est pris le jour même à l'unité médicale d'accueil de l'hôpital (UMA), localisation pratique car située juste à l'entrée de l'établissement; je n'allais pas au CMP ce jour-là. À la fin du rendez-vous, avant de quitter l'UMA, je vois le visage inquiet de l'interne de garde. La jeune femme m'appelle: «Pouvez-vous m'aider?» Le ton de la demande est pressant et le $\mathrm{PH}^{3}$ de l'urgence est justement absent pour quelques minutes, la relève infirmière est en train de se faire. L'interne m'explique qu'il s'agit d'un homme SDF de 45 ans qui exige une hospitalisation, alors que son psychiatre traitant a fait savoir qu'il n'y avait pas indication d'hospitalisation. Je reçois ce patient dans un bureau, avec l'interne. Je me présente, nom, titre et fonction, et je lui fais part du point de vue de son thérapeute. Il fait mine de casser l'écran d'ordinateur du bureau avec son front. Nous échangeons brièvement, puis il retourne dans la salle d'attente, debout, toujours énervé; l'excitation monte. J'essaie de rappeler 
son thérapeute sans pouvoir le joindre, et je pense en moi-même qu'il va peut être falloir appeler les gardes. Mais quelque chose se produit en moi, tout à fait inattendu: je me vois me diriger vers ce patient et m'asseoir à sa droite, sur le banc où il était assis; et je m'entends lui dire d'un air démuni bien réel: «On ne va tout de même pas appeler les gardes, ce serait nul.» Chose surprenante, dès que je m'assois à ses côtés, le patient se détend complètement dans son corps et un souffle s'écoule de sa bouche, comme lorsque l'on est soulagé après un fort stress. On aurait dit qu'il attendait quelqu'un à côté de lui. Il me répond avec conviction : «Oui, ce serait complètement nul.» Je lui dis alors que je sais qu'il est actuellement à la rue, qu'il n'apprécie sans doute pas les hébergements d'urgence, mais qu'en effet, son thérapeute estime qu'il n'y a pas d'indication d'hospitalisation, même si c'est bien dommage pour lui. Il se lève alors et dit, en hésitant, comme se parlant à lui-même : «Bon, alors, je pars, je vais me débrouiller. »

Il n'y a certes pas de quoi pavoiser d'avoir rendu à la rue et à son système un homme de la rue. Ma réflexion porte sur le geste qui m'est venu de m'asseoir à côté de lui, pleinement assumé, mais, pour ainsi dire, «sans le faire exprès ». [...] Qu'un professionnel puisse improviser juste (ou faux...) par le geste et la parole me paraît constituer un préalable à toute pratique de santé mentale. (Furtos, 2009, p. 14)

On voit, en l'occurrence, que le geste de s'asseoir sur le banc a été consécutif à un dialogue intérieur - « appeler les gardes? Oui ? Non ce serait nul.»dialogue qui est ensuite énoncé en une parole simple (Furtos, 2011). Le rapport conscient à l'intériorité, donc au non savoir, paraît important à supporter un temps. Par contre, par rapport aux procédures normatives, on sait qu'il est formellement déconseillé de s'assoir à côté d'un patient dont on redoute un passage à l'acte: ce que j'ai fait était donc une transgression des procédures, permission importante à observer par la jeune interne.

Dans ces types de situations, l'évaluation est dans le résultat, improbable au départ. Mais ne pas prendre le risque de l'improbable serait se comporter comme une machine triviale, c'est-à-dire entièrement programmée, entièrement procédurale, entièrement prévisible.

Un retour réflexif sur le quotidien permet de retrouver assez banalement la parole improvisée en français courant dans les actes prosaïques de la vie quotidienne, en institution, en psychothérapie, mais également en dehors de la clinique: avec les équipes, les collègues, les représentants de l'administration hospitalière et de l'État. Être et parler signifient dire quelque chose à 
quelqu'un, et pas n'importe quoi; le dire à quelqu'un signifie un réel engagement, par une parole destinée à un vrai autre, respecté en tant que ce qu'il est, dans une improvisation qui crée du neuf. À l'inverse, on connait le discours qui consiste à dire des choses que l'on sait déjà à quelqu'un qui n'en est pas surpris: on ouvre un tiroir et l'on croit parler au nom de la science, de la nosographie, de l'idéologie, de la morale. C'est quelquefois utile, mais pas tout le temps; rien ne se passe, on s'ennuie, on est dans le faux-self et le clivage. À l'extrême, le discours ne devient ni plus ni moins qu'une forme de mutisme bruyant, c'est-à-dire parler pour ne rien dire, le contraire de l'engagement. S'engager, c'est créer du neuf avec autrui sur la scène de l'intime comme sur celle du social, mais parler à autrui présuppose se parler à soimême, ce dialogue intérieur que Platon appelait l'âme: "Ainsi, penser et discourir, c'est tout un, à ceci près que ce que nous avons désigné du nom de pensée, c'est le dialogue intérieur et silencieux de l'âme avec elle-même.» (Sophiste, 263e, 264a)

\author{
Jean Furtos \\ Centre Hospitalier Le Vinatier \\ 95, boul. Pinel \\ 69677 Bron, France \\ jean.furtos@ch-le-vinatier.fr
}

\title{
Notes
}

1. Séminaire National de recherche clinique, organisé par l'ORSPERE (Observatoire Régional Rhône-Alpes sur la souffrance psychique en rapport avec l'exclusion), avec des fonds de la DGAS (Direction Générale de l'Action Sociale).

2. ORSPERE, décembre 1999, Points de vue et rôle des acteurs de la clinique psychosociale (recherche-action), ministère de l'Emploi et de la Solidarité.

3. Praticien hospitalier.

\section{Références}

DHERET, J., 2011, Créer les conditions de possibilité de la parole. Une dangereuse autonomie. Rhizome, 41, 6-8.

FREUD, S., 1929/1994, Malaise dans la culture. In Euvres complètes XVIII, Paris, PUF.

FURTOS, J., 2006, Transgression validée ou: de l'intelligence de l'action, Rhizome, 25, 55-57.

FURTOS, J., 2008, Les cliniques de la précarité - Contexte social, psychopathologie et dispositif, Paris, Masson.

FURTOS, J., 2009, De l'importance de l'interstice, Rhizome, 36, 14-15.

FURTOS, J., 2011, Parler en français courant, Rhizome, 41, 12-13.

LÉVINAS, E., 1978, Autrement qu'être ou au-delà de l'essence, La Haye, Martinus Nijhoff.

PLATON, 1989, Platon par lui-même, textes choisis et traduits par Louis Guillermit, Combas, Édition de l'éclat. 